\title{
Nerve growth factor enhances cough and airway obstruction via TrkA receptor- and TRPV1-dependent mechanisms
}

\author{
A Z El-Hashim, S M Jaffal
}

- Additional methods are published online only at http:// thorax.bmi.com/content/vol64/ issue9

Department of Applied Therapeutics, Faculty of Pharmacy, Kuwait University, Kuwait

Correspondence to: Dr A Z El-Hashim, Department of Applied Therapeutics, Faculty of Pharmacy, Kuwait University, PO Box 24923, Safat 13110, Kuwait; ahmed.elhashim@ hsc.edu.kw

Received 1 January 2009 Accepted 5 May 2009 Published Online First 3 June 2009

\begin{abstract}
Background: Nerve growth factor (NGF) is an important mediator of airway hyper-responsiveness and hyperalgesia but its role in cough is unknown.

Objectives: In this study the effects of NGF on the cough reflex and airway calibre were investigated in guinea pigs. The involvement of the tropomyosin-related kinase $A$ (TrkA) receptor and transient receptor potential vanilloid-1 (TRPV1), and the p38 mitogen-activated protein kinase (MAPK)-dependent pathway in any NGF-induced effects on cough and airway obstruction was also assessed. Methods: Guinea pigs were placed in a transparent whole-body plethysmograph box. Cough was assessed visually, acoustically and by analysis of the airflow signal. Airway obstruction was measured using enhanced pause (Penh) as an index.
\end{abstract}

Results: Exposure of guinea pigs to NGF did not induce a cough response nor a significant airway obstruction. However, exposure of guinea pigs to NGF immediately before citric acid inhalation resulted in a significant increase in the citric acid-induced cough and airway obstruction compared with vehicle-treated animals. Pretreatment with the TrkA receptor antagonist, K252a, or the TRPV1 antagonist, iodoresiniferatoxin, significantly inhibited the NGF-enhanced cough and airway obstruction. Exposure to NGF also increased p38 MAPK phosphorylation, but pretreatment with the p38 MAPK inhibitor, SB203580, did not affect either the NGFenhanced cough or airway obstruction despite preventing the NGF-induced elevation in p38 MAPK phosphorylation. Conclusions: The data show that NGF can enhance both cough and airway obstruction via a mechanism that involves the activation of the TrkA receptor and TRPV1 but not the p38 MAPK-dependent pathway.

Cough has a high prevalence rate and is one of the most common symptoms for which sufferers seek medical help from primary care physicians and pulmonologists. ${ }^{1}$

Several pharmacological receptors have been identified to be important in mediating cough. ${ }^{2} 3$ Currently, however, most of the mechanistic information on cough is essentially derived from animal models with a normal cough reflex where a baseline-type cough is induced through the use of chemicals such as citric acid or capsaicin.. ${ }^{45}$ Although this approach is very useful in dissecting out the airway cough receptors and those gating the cough centre, we also need to address major issues with some types of clinical cough such as the enhanced cough reflex which is seen in acute cough associated with upper respiratory tract infections (URTIs). ${ }^{6}$ Hence, mediators involved in lowering the cough threshold and the mechanisms involved need to be studied. Models of chronic spontaneous cough associated with URTI, gastro-oesophageal reflux and asthma are also needed to improve the understanding of the mechanisms underlying this type of cough.

Nerve growth factor (NGF) is a member of the neurotrophin family of proteins that are known to regulate neuronal development and maintenance, and are also involved in the sensitisation of spinal circuitry that underlies many forms of hyperalgesia. The biological activity of NGF is thought to be mediated by two main receptors: the high affinity tropomyosin-related kinase A (TrkA) receptor containing an intrinsic tyrosine kinase activity and a low affinity receptor p75 for neurotrophins (NTRs). ${ }^{8}$

The notion that NGF may be an important mediator of enhanced cough is implied by studies highlighting its importance in airway hyperresponsiveness and hyperalgesia; two phenomena that share many similarities with cough. ${ }^{10}$ For example, NGF has been shown to induce airway hyper-responsiveness in vivo in guinea pigs $^{11}$ and mice, ${ }^{12}{ }^{13}$ and in vitro in human bronchus. ${ }^{14}$ Also, NGF was reported to be an important mediator in both primary and secondary hyperalgesia. ${ }^{15} 16$ Although both NGF receptors appear to have a role in hyperalgesia, there is stronger evidence for TrkA receptor involvement. ${ }^{17}$ There is also evidence to suggest that NGF sensitisation of nociceptive neurons is mediated via the activation of transient receptor potential vanilloid-1 (TRPV1) ${ }^{18}$ and that both p38 mitogen-activated protein kinase (MAPK)- and phosphatidylinositol 3-kinase (PI3K)-dependent pathways seem to couple the activation of TrkA receptors to TRPV1 stimulation. ${ }^{18}$ A recent clinical study did not find a significant increase in NGF levels in either serum or sputum in patients with chronic cough. However, it did report a significant correlation between the duration of cough and NGF sputum levels, suggesting a complex relationship between cough and NGF. ${ }^{20}$ NGF levels have also been reported to be elevated in asthma ${ }^{21}$ and in allergic rhinitis following allergen provocation, ${ }^{22}$ suggesting a possible role for this mediator in some aspects of respiratory disease.

In order to study the effect of NGF on the cough reflex and airway calibre we investigated (1) whether NGF can enhance the citric acid-induced cough and airway obstruction and (2) the role of TrkA receptors and TRPV1 channels as well as the p38 MAPKdependent pathway in any NGF-mediated effects on the cough reflex and airway obstruction. 


\section{A}

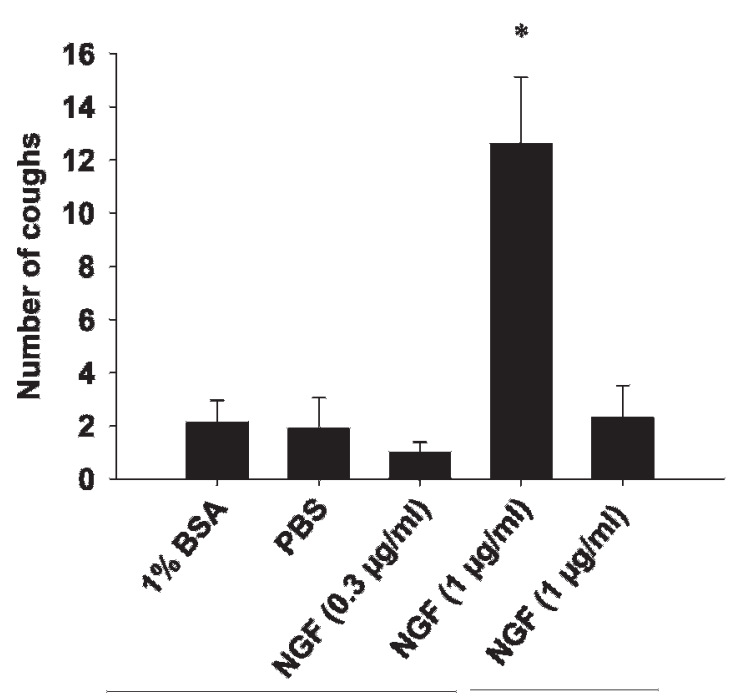

Citric acid challenge, immediate

Citric acid challenge, post $24 \mathrm{hr}$

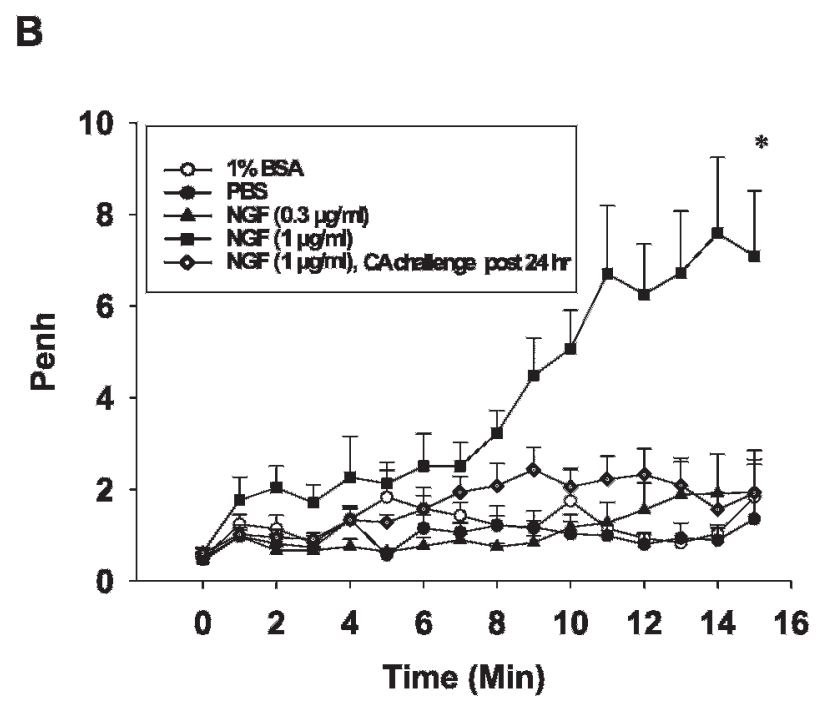

Figure 1 Effect of nerve growth factor (NGF) treatment both immediately $(0.3$ and $1 \mu \mathrm{g} / \mathrm{ml})$ and after $24 \mathrm{~h}(1 \mu \mathrm{g} / \mathrm{ml})$ on citric acidinduced cough (A) and airway obstruction (B) in guinea pigs. Values are presented as the mean (SEM). * Statistically significant difference $(p<0.05)$ compared with phosphate-buffered saline $(P B S)-$, bovine serum albumin (BSA)-, $0.3 \mu \mathrm{g} / \mathrm{ml}$ (immediate) and $1 \mu \mathrm{g} / \mathrm{ml}$ (post $24 \mathrm{~h}$ ) NGF-treated animals. Penh, enhanced pause.

\section{METHODS}

\section{Animals}

Dunkin-Hartley guinea pigs (300-600 g) of both sexes were used in this study. The care and use of animals was approved by the Animal Welfare and Use of Laboratory Animals Committee in the Health Sciences Center, Kuwait University. All animals were randomly assigned to control and experimental groups.

\section{Measurement of cough response}

The animals were continuously watched by a trained observer who counted the number of coughs. Coughs were recognised by the characteristic animal posture and a rapid transient increase in airflow over and above the normal flow. Also, the criteria for cough included a high sound with the mouth open and a
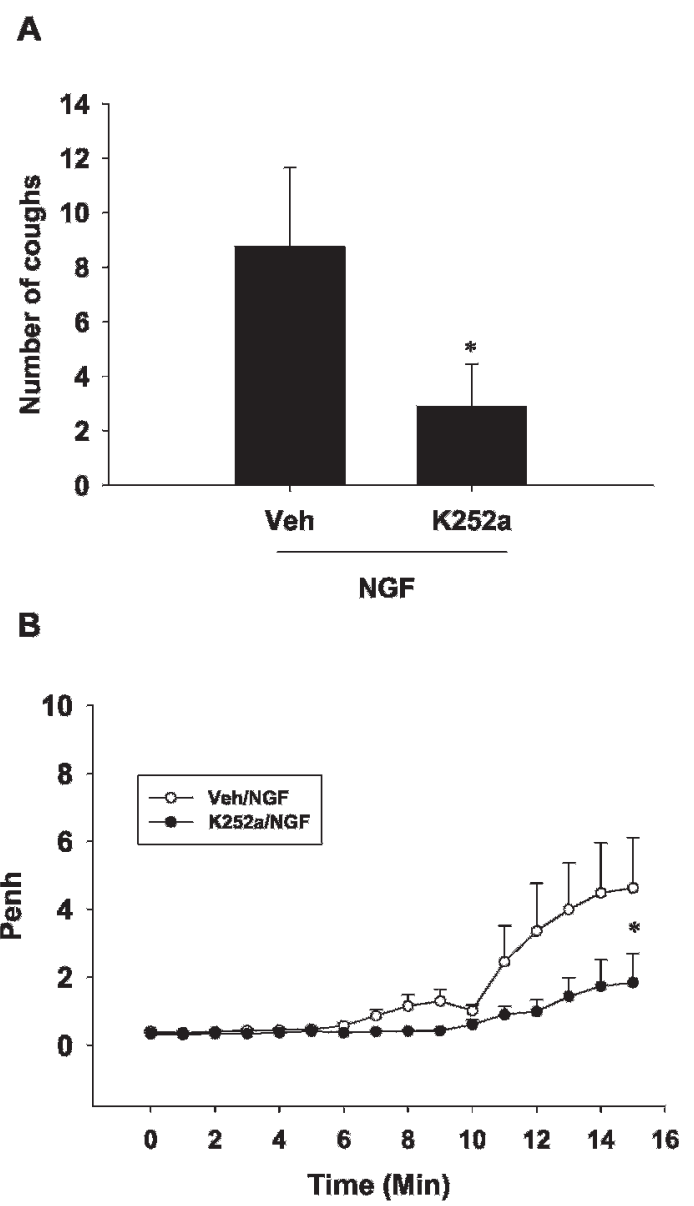

Figure 2 Effect of $\mathrm{K} 252 \mathrm{a}(20 \mu \mathrm{g} / \mathrm{kg}$; intraperitoneally) on nerve growth factor (NGF)-enhanced citric acid-induced cough (A) and airway obstruction (B) in guinea pigs. Values are presented as the mean (SEM). ${ }^{*}$ Statistically significant difference $(p<0.05)$ compared with vehicle (Veh)-treated animals. Penh, enhanced pause.

defined pattern in the sound signal which distinguishes coughs from sneezes.

\section{Measurement of airway obstruction}

Airway function was measured using whole-body plethysmography. For more details refer to the additional methods online.

Protein isolation from the lungs and western blot analysis For details refer to the additional methods online.

\section{Compounds}

Chemicals used were citric acid, phosphate-buffered saline (PBS) tablets, bovine serum albumin (BSA), SB203580, nerve growth factor (NGF-7S mouse submaxillary glands) and $\alpha$-actin monoclonal antibody (Sigma-Aldrich, Taufkirchen, Germany), K252a (Fluka BioChemika, Buschs, Switzerland), 5' iodoresiniferatoxin (IRTX) (Tocris, Cookson, Langford, UK), dimethylsulfoxide (DMSO) (BDH Laboratory Supplies, Poole, UK), nitrocellulose membranes (Biorad Laboratories, Hercules, California, USA), an ECL (enhanced chemiluminescence) kit, and primary and secondary antibodies (Amersham, Little Chalfont, UK). Stock solutions of NGF $(50 \mu \mathrm{g} / \mathrm{ml})$ in $1 \%$ BSA in PBS were stored in aliquots at $-80^{\circ} \mathrm{C}$ and used as freshly prepared dilutions of 0.3 and $1 \mu \mathrm{g} / \mathrm{ml}$. Stock solution of K252a 
A

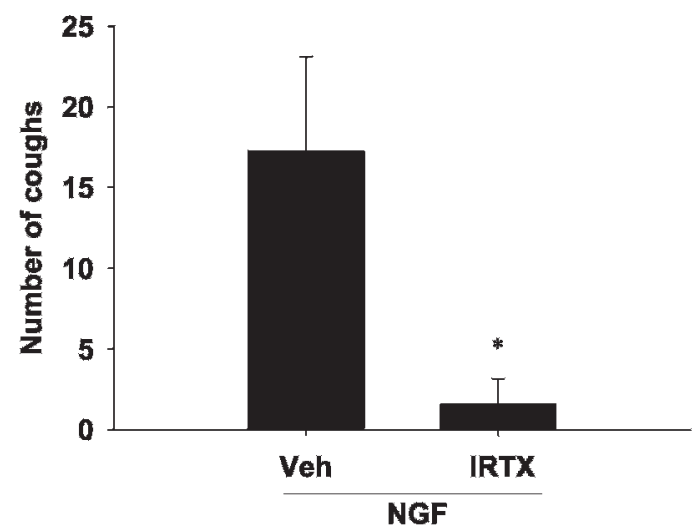

B

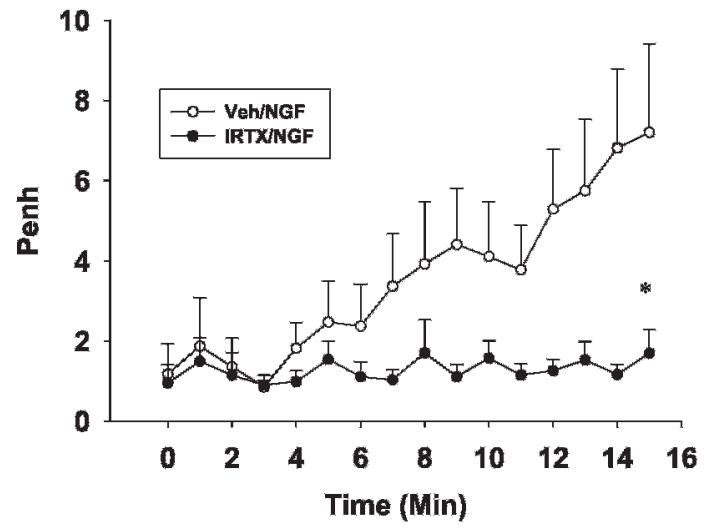

Figure 3 Effect of $5^{\prime}$ iodoresiniferatoxin (IRTX; $7.5 \mu \mathrm{g} / \mathrm{ml}$ ) on nerve growth factor (NGF)-enhanced citric acid-induced cough (A) and airway obstruction (B) in guinea pigs. Values are presented as the mean (SEM). * Statistically significant difference $(p<0.05)$ compared with vehicle (Veh)-treated animals. Penh, enhanced pause.

was made by dissolution in 30\% DMSO (in normal saline); subsequent dilutions were made in normal saline. Stock solutions of IRTX and SB203580 were made by dissolution in $100 \%$ DMSO; subsequent dilutions were made in distilled water. All the stock solutions were kept in aliquots at $-80^{\circ} \mathrm{C}$ and dilutions were made on the day of the experiment. Citric acid (0.2 M) was freshly prepared in PBS.

\section{Experimental protocol}

Effect of immediate and $24 \mathrm{~h}$ post-NGF aerosolisation on citric acidinduced cough and airway obstruction in guinea pigs

Five groups were randomly assigned. Groups $1(n=10)$ and 2 $(\mathrm{n}=14)$ received NGF (0.3 and $1 \mu \mathrm{g} / \mathrm{ml}$, respectively) by inhalation over $10 \mathrm{~min}$ and were then immediately given $0.2 \mathrm{M}$ inhaled citric acid. Two control groups (3 and 4) were set up. Group $3(n=14)$ received BSA (in PBS) which was the vehicle for NGF. Group $4(n=10)$ received PBS which was the vehicle for BSA (the latter group was established so as to ascertain whether BSA had any effect on cough or airway calibre). Immediately following exposure to either BSA or PBS, animals were given $0.2 \mathrm{M}$ inhaled citric acid. Group $5(\mathrm{n}=10)$ received NGF $(1 \mu \mathrm{g} / \mathrm{ml})$ and $24 \mathrm{~h}$ later the animals were given $0.2 \mathrm{M}$ inhaled citric acid. Cough and airway obstruction were assessed in all groups during the citric acid challenge and $5 \mathrm{~min}$ thereafter.
A
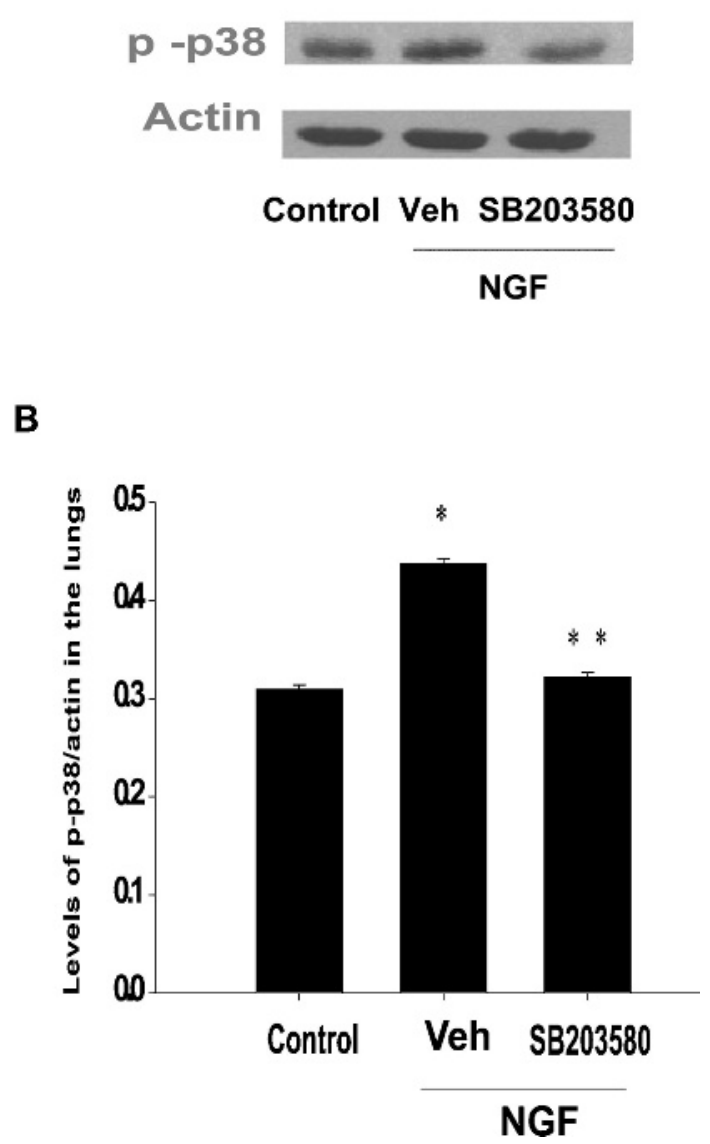

Figure 4 (A) Western blot analysis of phosphorylated p38 mitogenactivated protein kinase (MAPK) protein levels from lungs of guinea pigs exposed to bovine serum albumin (BSA) (control), by the inhaled route. The other two groups were initially pretreated with either vehicle (Veh) or SB203580-pretreated guinea pigs and exposed to nerve growth factor (NGF). (B) Histogram of western blots showing the NGF-induced elevation in p38 MAPK phosphorylation and subsequent reduction after SB203580 pretreatment. Data shown represent the mean (SEM) p38 phosphorylation intensity relative to actin $(n=3)$. * Statistically significant difference $(p<0.05)$ compared with BSA-exposed animals. ${ }^{* *}$ Statistically significant difference $(p<0.05)$ compared with vehicletreated animals.

Effect of the TrkA receptor antagonist, K252a $(20 \mu \mathrm{g} / \mathrm{kg})$, on NGFenhanced citric acid-induced cough and airway obstruction

Two groups were randomly assigned. Group $1(n=8)$ was pretreated with the vehicle and group $2(\mathrm{n}=9)$ was pretreated with K252a (20 $\mu \mathrm{g} / \mathrm{kg}$, intraperitoneally). A dose of $20 \mu \mathrm{g} / \mathrm{kg}$ of the inhibitor K252a was chosen as a similar dose was previously reported to significantly inhibit the development of anaphylactic shock in guinea pigs. ${ }^{23}$ After $30 \mathrm{~min}$, animals from both groups received inhaled NGF $(1 \mu \mathrm{g} / \mathrm{ml})$ for $10 \mathrm{~min}$. Immediately following NGF exposure, animals were exposed to inhaled citric acid $(0.2 \mathrm{M})$ for $10 \mathrm{~min}$. Both cough and airway obstruction were assessed during the citric acid challenge and $5 \mathrm{~min}$ thereafter.

Effect of the TRPV1 antagonist, IRTX $(7.5 \mu \mathrm{g} / \mathrm{ml})$, on NGF-enhanced citric acid-induced cough and airway obstruction

Two groups were randomly assigned. Group $1(n=8)$ was pretreated with the vehicle and group $2(n=9)$ was pretreated 
A

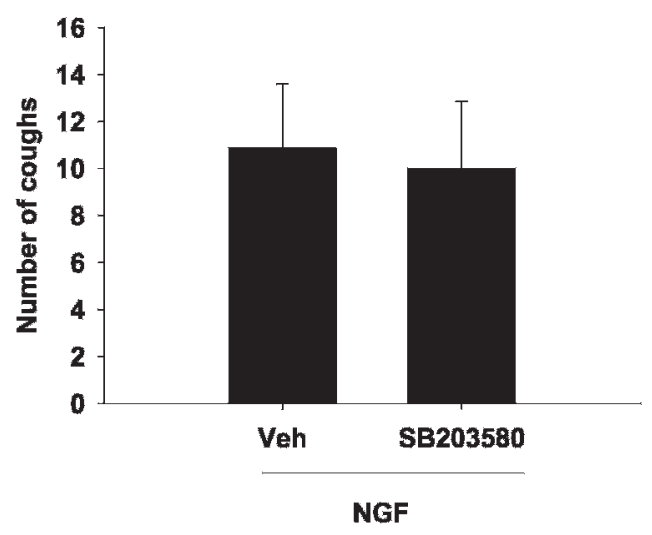

B

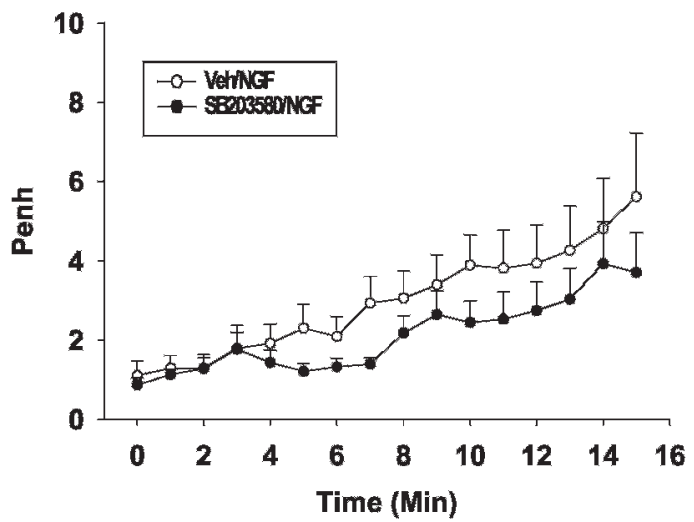

Figure 5 Effect of SB203580 $(22.6 \mu \mathrm{g} / \mathrm{ml})$ on nerve growth factor (NGF)-enhanced citric acid-induced cough (A) and airway obstruction (B) in guinea pigs. Values are presented as mean (SEM). Penh, enhanced pause; Veh, vehicle.

with IRTX $(7.5 \mu \mathrm{g} / \mathrm{ml})$. A dose of $7.5 \mu \mathrm{g} / \mathrm{ml}$ IRTX was chosen as a similar dose was previously reported to have an inhibitory effects on cough. ${ }^{4}$ Both vehicle and drug were administered by aerosol over $10 \mathrm{~min}$. Immediately following exposure to IRTX or its vehicle, the animals received inhaled NGF $(1 \mu \mathrm{g} / \mathrm{ml})$ for $10 \mathrm{~min}$. At the end of the NGF exposure, the animals were exposed to inhaled citric acid $(0.2 \mathrm{M})$ for $10 \mathrm{~min}$. Both cough and airway obstruction were assessed during the citric acid challenge and $5 \mathrm{~min}$ thereafter.

Effect of the p38 MAPK inhibitor, SB203580 $(22.6 \mu \mathrm{g} / \mathrm{ml})$, on NGFenhanced citric acid-induced cough and airway obstruction

Two groups were randomly assigned. Group $1(n=8)$ was pretreated with the vehicle and group $2(\mathrm{n}=9)$ was pretreated with SB203580 $(22.6 \mu \mathrm{g} / \mathrm{ml})$. This dose was based on a study where SB203580 was shown to inhibit NGF-induced phosphorylation of $\mathrm{p} 38 \mathrm{MAPK} \cdot{ }^{18}$ Both vehicle and drug were given by aerosol over $10 \mathrm{~min}$. Immediately following exposure to SB203580 or its vehicle, the animals received inhaled NGF $(1 \mu \mathrm{g} / \mathrm{ml})$ for $10 \mathrm{~min}$. At the end of the NGF exposure, the animals were exposed to inhaled citric acid (0.2 M) for $10 \mathrm{~min}$. Both cough and airway obstruction were assessed during the citric acid challenge and 5 min thereafter.

For the protocols using two treatment groups, the experiment was typically run twice or three times with $n=2-3$ guinea pigs in each treatment group each time. For the protocol using five treatment groups, the experiment was typically run $3-5$ times with $n=2-3$ guinea pigs in each treatment group each time. This ensured that all groups were subjected to the same conditions at the same time and hence reduced variability. Also, all groups had an almost equal number of male and female guinea pigs.

\section{Expression of results and statistical analysis}

For the cough experiments, the data are expressed as mean (SEM) and represent the number of coughs during a $15 \mathrm{~min}$ period. Airway obstruction is represented as an absolute change in Penh and is expressed as the mean (SEM). The differences in the degree of the airway obstruction between different groups were determined by calculating the mean Penh value for the last $10 \mathrm{~min}$ for each guinea pig. All differences between groups were expressed as the mean difference. All the data were first analysed for normal distribution then tested for statistical difference using, in the case of the multiple groups, a one-way analysis of variance (ANOVA). The difference between two treatment groups was analysed using either a parametric (non-paired t test) or nonparametric test (Mann-Whitney rank sum test). All statistical analyses were carried out using a statistical program (SPSS version 17; SPSS, Chicago, Illinois, USA) and for all studies probability values of $p<0.05$ were considered statistically significant.

\section{RESULTS}

Effect of immediate and $24 \mathrm{~h}$ post-NGF treatment on citric acidinduced cough and airway obstruction in guinea pigs

During the NGF exposure (either 0.3 or $1 \mu \mathrm{g} / \mathrm{ml}$ ), no cough response or statistically significant airway obstruction was noted (data not shown). However, approximately $40 \%$ of the guinea pigs exposed to NGF $(1 \mu \mathrm{g} / \mathrm{ml})$ did demonstrate a modest degree of airway obstruction compared with BSA- or PBS-exposed animals (data not shown). Our data show that the citric acid-induced cough in the PBS-pretreated animals was low, with a mean number of 1.9 (1.2) coughs over $10 \mathrm{~min}$. Pretreatment with NGF aerosol, at $1 \mu \mathrm{g} / \mathrm{ml}$ but not $0.3 \mu \mathrm{g} / \mathrm{ml}$, induced a statistically significant increase in the mean number of coughs compared with PBS-exposed animals, with a mean difference of $10.70(p<0.001)$. This increase in the mean number of coughs was specifically due to the NGF and not the BSA as administration of BSA alone resulted in a cough response similar to that seen in the PBS-treated group, with a mean difference of $0.23(p=0.92)$. Indeed, NGF-enhanced cough was statistically significantly greater than in both the PBS- and BSA-treated groups (fig 1A). Furthermore, no enhanced cough was observed $24 \mathrm{~h}$ following exposure to $1 \mu \mathrm{g} / \mathrm{ml}$ NGF compared with the PBS group; the mean difference was $0.40(p=0.87)$. On the basis of these studies, $1 \mu \mathrm{g} / \mathrm{ml}$ of NGF was selected for subsequent experiments.

Our data also show that the citric acid-induced airway obstruction in the PBS-pretreated animals was low, with a mean Penh value of 0.9 (0.1). Pretreatment with NGF aerosol, at $1 \mu \mathrm{g} / \mathrm{ml}$ but not $0.3 \mu \mathrm{g} / \mathrm{ml}$, induced a statistically significant increase in the citric acid-induced airway obstruction compared with PBS-treated guinea pigs, with a mean difference of 4.28 $(p<0.001$, fig 1B). This increase in the airway obstruction again was specific to NGF and not BSA as administration of BSA alone did not result in a significant airway obstruction compared with that seen in the PBS-treated group; the mean difference was $0.36(p=0.65$, fig $1 B)$. Indeed, the NGF- $(1 \mu \mathrm{g} /$ $\mathrm{ml}$ ) enhanced airway obstruction was statistically significantly greater than in both the PBS- and BSA-treated groups, with a mean difference of $4.28 \quad(p<0.001)$ and $3.92 \quad(p<0.001)$, 
respectively. Additionally, the NGF- $(1 \mu \mathrm{g} / \mathrm{ml})$ enhanced airway obstruction had declined by $24 \mathrm{~h}$ following NGF treatment and was not significantly different from that of the PBS-exposed group; the mean Penh difference was 1.10 ( $p=0.22$, fig $1 \mathrm{~B}$ ).

\section{Effect of K252a on NGF-enhanced citric acid-induced cough and airway obstruction}

Studies have shown that the hyperalgesic effect of NGF is mediated via TrkA receptors. Therefore, we assessed the involvement of the TrkA receptor in NGF-enhanced citric acid-induced cough and airway obstruction. Pretreatment of guinea pigs with $\mathrm{K} 252 \mathrm{a}(\mathrm{n}=9)$ induced a statistically significant inhibition of the NGF-enhanced citric acid-induced cough compared with vehicle- $(n=8)$ pretreated guinea pigs; the mean difference was 5.86 ( $p=0.046$, fig 2A). Moreover, pretreatment with $\mathrm{K} 252 \mathrm{a}$ resulted in a statistically significant reduction in the NGF-enhanced citric acid-induced airway obstruction compared with vehicle-pretreated animals; the mean difference was 1.47 ( $p=0.021$, fig $2 B$ ).

\section{Effect of IRTX on NGF-enhanced citric acid-induced cough and airway obstruction}

The involvement of TRPV1 in mediating the effects of NGFenhanced citric acid-induced cough and airway obstruction was investigated. Pretreatment of guinea pigs with IRTX $(n=9)$ induced a statistically significant reduction in the NGF-enhanced citric acid-induced cough compared with vehicle-pretreated $(n=8)$ guinea pigs; the mean difference was 15.68 ( $p=0.007$, fig 3A). Moreover, pretreatment with IRTX resulted in a statistically significant inhibition of the NGF-enhanced citric acid-induced airway obstruction compared with vehicle-pretreated animals; the mean difference was 3.37 ( $p=0.049$, fig 3B).

\section{Effect of NGF and pretreatment with SB203580 on p38 MAPK levels in the lungs}

To assess if the link between the activation of TrkA receptors and that of TRPV1 channels is mediated via a p38 MAPKdependent pathway, its phosphorylation following NGF treatment was investigated. Using a phospho-specific p38 antibody, we were able to detect basal expression of p38 MAPK in the lungs of control animals - that is, BSA-treated guinea pigs (fig 4A,B). NGF treatment induced a modest but statistically significant elevation in the levels of p38 MAPK phosphorylation in the lungs $(p<0.001)$. Moreover, the NGF-induced p38 MAPK phosphorylation was prevented following pretreatment with SB203580 ( $<<0.001$; fig 4A,B).

\section{Effect of SB203580 on NGF-enhanced citric acid-induced cough and airway obstruction}

These experiments were conducted to investigate if the MAPK inhibitor, SB203580, has an effect on the NGF-enhanced citric acid-induced cough and airway obstruction. Pretreatment of guinea pigs with SB203580 $(22.6 \mu \mathrm{g} / \mathrm{ml} ; \mathrm{n}=9)$ did not significantly affect the NGF-enhanced citric acid-induced cough when compared with vehicle-pretreated $(n=8)$ guinea pigs; the mean difference was $0.85(p=0.83$, fig $5 \mathrm{~A})$. Also, pretreatment with SB203580 did not significantly affect the NGF-enhanced citric acid-induced airway obstruction compared with vehicle-pretreated animals; the mean difference was 1.19 ( $p=0.27$, fig $5 B$ ).

\section{DISCUSSION}

The findings of this study show that NGF is not a tussigenic mediator. However, pre-exposure of guinea pigs to NGF significantly increases both the citric acid-induced cough and airway obstruction. This effect is seen within minutes of NGF exposure but was completely resolved after $24 \mathrm{~h}$. Additionally, the data show that NGF-induced enhancement of cough and airway obstruction is mediated predominantly via the TrkA receptor as treatment with $\mathrm{K} 252 \mathrm{a}$ significantly reduced both these effects. Furthermore, pretreatment with IRTX also significantly reduced both the NGF enhancement of cough and airway obstruction, suggesting an important role for TRPV1 in mediating the NGF enhancement of airway responses. Our data also show that NGF increases the phosphorylation of p38 MAPK in the lungs and this effect was significantly inhibited by the p38 MAPK inhibitor, SB203580. However, SB203580 did not inhibit the NGFmediated enhancement of either cough or airway obstruction, suggesting that whilst the p38 MAPK-dependent pathway is activated in the lungs by NGF, it is not critical for the enhancement of these airway responses.

We selected the dose of $0.2 \mathrm{M}$ citric acid on the basis of previous observations that this dose is a low grade stimulus for both cough and airway obstruction. ${ }^{24}$ It was considered important to use a low dose of citric acid so that any NGF enhancment effects, on either cough or airway obstruction, would be more easily discernible. Additionally, as some studies have reported that IRTX can inhibit citric acid-induced baseline cough, ${ }^{4}$ it was important to ensure that any TRPV1 involvement was mainly NGF dependent.

Exposure of guinea pigs to NGF $(0.3$ and $1 \mu \mathrm{g} / \mathrm{ml})$ had no direct effect on the cough reflex nor did NGF induce a statistically significant airway obstruction. Although it is possible that higher doses may have induced a cough response, this is unlikely as the $1 \mu \mathrm{g} / \mathrm{ml}$ dose induced some degree of airway obstruction in some animals yet those animals did not cough. Our data also show that NGF enhances the cough response to citric acid challenge. To the best of our knowledge this is the first report showing that NGF sensitises the cough reflex. This effect was evident with $1 \mu \mathrm{g} / \mathrm{ml}$ but not the lower dose $(0.3 \mu \mathrm{g} / \mathrm{ml})$ and had disappeared by $24 \mathrm{~h}$, suggesting that an acute exposure to NGF induces only an acute effect. This effect of NGF on the cough reflex is in agreement with findings in models of pain which demonstrate an important role for NGF in hyperalgesia. For example, both animal and human studies have shown that administration of NGF causes a robust mechanical and thermal hyperalgesia following either local or systemic administration. ${ }^{25-27}$ Moreover, the time frame for the development of NGF-induced hyperalgesic effects was similar to what we have observed in the present study-that is, $<30$ min. ${ }^{25} 26$ Interestingly, however, whereas chronic cough appears to be associated with inflammation, neurotrophins, including NGF, do not appear to be elevated. ${ }^{20}$ However, a strong correlation between sputum NGF levels and the duration of cough exists. $^{20}$ Therefore, the role of NGF in both human chronic and acute cough associated with URTI still remains to be elucidated.

Our results show that airway obstruction to citric acid was also significantly enhanced following NGF pretreatment. This finding is in agreement with previous data showing that NGF enhances the contractile responses of guinea pig tracheal strips to histamine and also in vivo airway obstruction to both histamine and neurokinin $A^{28-30}$ The mechanism by which NGF may mediate this effect is postulated to be via both enhancement of neuropeptide release and induction of their synthesis, ${ }^{11}{ }^{28} 29$ and also possibly via a histamine receptor-dependent pathway. ${ }^{30}$

The biological activity of NGF is mediated by two receptors: the high affinity TrkA receptor and the low affinity p75 NTR 
receptor. ${ }^{8}$ In contrast to p75 NTR which binds to all neurotrophins such as NGF, brain-derived neurotrophin (BDNF) and neurotrophin-3/4/5 (NT-3/4/5) with equal affinities, the TrkA receptor is more selective for NGF. ${ }^{14}{ }^{31}$ Because of the higher selectivity of the TrkA receptor for NGF and the fact that this receptor has been associated with the effects of NGF in hyperalgesia and inflammation, ${ }^{19}{ }^{32}$ we investigated the role of TrkA receptors in mediating the NGF enhancement of cough and airway obstruction. Our data show that TrkA receptors play a central role in mediating the NGF-induced sensitisation of airway effects as inhibition of this receptor with K252a significantly reduced both the NGF-enhanced cough and airway obstruction. This is in agreement with studies showing that either neutralising TrkA receptors with monoclonocal antibodies or inhibiting them with K252a inhibits the NGFinduced hyperalgesia. ${ }^{33}{ }^{34}$ Therefore, our data are a further confirmation of the importance of this receptor in sensitising sensory nerves. However, recent evidence has also suggested a role for the p75 NTR in NGF-mediated sensitisation of sensory nerves, ${ }^{35}$ and hence a role for this receptor in these airway responses remains to be established.

The heat-gated ion channel TRPV1 is an important detector of noxious levels of heat, and evidence is accumulating to suggest that this receptor appears to play a critical role in hyperalgesia. ${ }^{36}$ Hence, the role of TRPV1 in mediating the NGFenhanced cough and airway obstruction was investigated. Interestingly, we observed that the TRPV1 antagonist, IRTX, very effectively inhibited both the NGF-enhanced cough and airway obstruction. Although it has been previously reported that TRPV1 channels are partly implicated in the baseline citric acid-induced cough, ${ }^{4}$ baseline cough was negligible in our study and hence it can be strongly argued that the effects of IRTX are due to the inhibition of the NGF enhancement of airway effects. This finding also fits in with data obtained in several studies. For example, in culture, NGF rapidly potentiates the activity of TRPV1 channels in DRG neurons treated with capsaicin. ${ }^{37}$ Zhang and co-workers ${ }^{19}$ have also reported that NGF enhances TRPV1 function, which was associated with TRPV1 phosphorylation, and also their increased membrane expression. Furthermore, a recent study has provided convincing data showing that NGF sensitises TRPV1-mediated signalling and that this was not via an increase in the unitary conductance or activation of quiescent channels but rather through an increase in the total number of plasma membrane TRPV1 channels. ${ }^{38}$ It is also noteworthy that these NGF-induced effects in both studies were observed within minutes - that is, a rapid effect which is in accordance with the time frame of the responses that were noted in our experiments.

In addition to confirming the involvement of TRPV1 in the NGF enhancement of cough and airway obstruction, we investigated the possible biochemical pathway linking TrkA receptors and TRPV1s, which thus far remains controversial, with several candidate signalling pathways proposed. ${ }^{18} 19$ 38-40

TrkA receptor signalling is currently thought to be mediated by three signalling pathways which include p38 MAPK, PI3K and phospholipase C $\gamma$ (PLC $\gamma) .{ }^{18} 3940$ Our data show that basal levels of phosphorylated p38 MAPK are indeed modestly increased following NGF treatment. This evidence would suggest that the NGF-TrkA signalling is, at least partly, mediated via the p38 MAPK-dependent pathway. Moreover, the p38 MAPK inhibitor, SB203580, significantly reduced the NGF-induced p38 MAPK phosphorylation. Surprisingly, however, our data showed that SB203580 did not affect the NGF-enhanced cough or airway obstruction. This suggests that the p38
MAPK-dependent pathway, although activated by NGF, does not seem to be involved in the enhancement of cough or airway obstruction by NGF. An alternative paradigm would be via the PI3K and PLC $\gamma$ pathways. Indeed, evidence exists showing that these signalling pathways are activated by NGF and can enhance TRPV1 expression. ${ }^{38-40}$ Hence, the involvement of these pathways in NGF-enhanced cough and airway obstruction needs to be investigated. It is also important to mention that several other mediators such as prostaglandin $\mathrm{E}_{2}$ and histamine ${ }^{30} 4142$ have been implicated in the signalling of NGF-mediated airway responses and hence their involvement in the enhancement of cough and airway obstruction cannot be ruled out. In our study, the location of the TrkA receptors was not addressed; however, they are known to be expressed in non-neuronal as well as neuronal sites. ${ }^{43-46}$ Also, a central site of action for NGF in enhancing cough is likely and remains to be determined.

In summary, our data show that although NGF does not directly induce cough or significant airway obstruction, it significantly enhances both the citric acid-induced cough and airway obstruction. These effects are mediated via both the TrkA receptor and TRPV1, and although NGF activates p38 MAPK in the lungs, this pathway does not appear to be involved in mediating the NGF enhancement of cough or airway obstruction. Our findings would suggest that the TrkA receptor may be an attractive drug target for inhibiting enhanced cough without affecting the normal cough reflex.

Acknowledgements: We are very grateful to Professor Saghir Akhtar for performing the western blot experiments. We would like to thank Dr Abdelmoneim Awad and Professor Yunus Luqmani for their constructive comments on the manuscript and also Heba Abdou for her initial technical work on this project. We also acknowledge the support of Ola Zahran and Sunny Okojo from the animal resource centre.

Funding: This study was funded by Kuwait University Research Administration grant number PT01/05.

\section{Competing interests: None.}

Ethics approval: The study was approved by the Animal Welfare and Use of Laboratory Animals' Committee at the Health Sciences Center, Kuwait University.

Provenance and peer review: Not commissioned; externally peer reviewed.

\section{REFERENCES}

1. Morice AH, McGarvey L, Pavord I, et al. Recommendations for the management of cough in adults. Thorax 2006;61:i1-24.

2. Bolser DC. Central mechanisms II: pharmacology of brainstem pathways. Handb Exp Pharmacol 2009;187:203-17

3. Canning BJ, Mori N, Mazzone SB. Vagal afferent nerves regulating the cough reflex. Respir Physiol Neurobiol 2006;152:223-42.

4. Trevisani M, Milan A, Gatti $\mathrm{R}$, et al. Antitussive activity of iodo-resiniferatoxin in guinea pigs. Thorax 2004;59:769-72.

5. El-Hashim AZ, Amine SA. The role of substance $P$ and bradykinin in the cough reflex and bronchoconstriction in guinea-pigs. Eur J Pharmacol 2005;513:125-33.

6. Eccles R, Lee PC. Cough induced by airway vibration as a model of airway hyperreactivity in patients with acute upper respiratory tract infection. Pulm Pharmacol Ther 2004;17:337-42.

7. Freund-Michel V, Frossard N. The nerve growth factor and its receptors in airway inflammatory diseases. Pharmacol Ther 2008;117:52-76.

8. Hoyle GW. Neurotrophins and lung disease. Cytokine Growth Factor Rev 2003:14:551-8.

9. Karlsson JA. A role for capsaicin sensitive, tachykinin containing nerves in chronic coughing and sneezing but not in asthma: a hypothesis. Thorax 1993;48:396-400.

10. Mazzone SB, Mori N, Canning BJ. Synergistic interactions between airway afferent nerve subtypes regulating the cough reflex in guinea-pigs. J Physiol 2005;569:559-73

11. de Vries A, Dessing MC, Engels F, et al. Nerve growth factor induces a neurokinin-1 receptor-mediated airway hyperresponsiveness in guinea pigs. Am J Respir Crit Care Med 1999;159:1541-4.

12. Braun A, Appel E, Baruch $R$, et al. Role of nerve growth factor in a mouse model of allergic airway inflammation and asthma. Eur J Immunol 1998;28:3240-51.

13. Braun A, Quarcoo D, Schulte-Herbrüggen 0 , et al. Nerve growth factor induces airway hyperresponsiveness in mice. Int Arch Allergy Immunol 2001;124:205-7.

14. Frossard N, Naline E, Olgart Höglund C, et al. Nerve growth factor is released by IL1 beta and induces hyperresponsiveness of the human isolated bronchus. Eur Respir $\mathrm{J}$ 2005; 26:15-20. 
15. Summer GJ, Puntillo KA, Miaskowski C, et al. TrkA and PKC-epsilon in thermal burninduced mechanical hyperalgesia in the rat. J Pain 2006;7:884-91.

16. Hathway GJ, Fitzgerald M. Time course and dose-dependence of nerve growth factor-induced secondary hyperalgesia in the mouse. J Pain 2006;7:57-61.

17. Watson JJ, Allen SJ, Dawbarn D. Targeting nerve growth factor in pain: what is the therapeutic potential?. BioDrugs 2008;22:349-59.

18. Ji RR, Samad TA, Jin SX, et al. p38 MAPK activation by NGF in primary sensory neurons after inflammation increases TRPV1 levels and maintains heat hyperalgesia. Neuron 2002;36:57-68.

19. Zhang $\mathbf{X}$, Huang J, McNaughton PA. NGF rapidly increases membrane expression of TRPV1 heat-gated ion channels. EMBO J 2005;24:4211-23.

20. Chaudhuri R, McMahon AD, McSharry CP, et al. Serum and sputum neurotrophin levels in chronic persistent cough. Clin Exp Allergy 2005;35:949-53.

21. Raap U, Wardlaw AJ. A new paradigm of eosinophil granulocytes: neuroimmune interactions. Exp Dermatol 2008;17:731-8.

22. Bonini S, Lambiase A, Bonini S, et al. Circulating nerve growth factor levels are increased in humans with allergic diseases and asthma. Proc Natl Acad Sci USA 1996;93:10955-60.

23. Paz 0, Ashkenazy Y, Moshonov S, et al. Attenuation of anaphylactic shock and related mortality in guinea-pigs after administration of a potent protein kinase inhibitor, K252a. J Basic Clin Physiol Pharmacol 1991;2:287-95.

24. El-Hashim AZ, Wyss D, Lewis C. Effect of a novel NK1 receptor selective antagonist (NKP608) on citric acid induced cough and airway obstruction. Pulm Pharmacol Ther 2004;17:11-8.

25. Lewin GR, Rueff A, Mendell LM. Peripheral and central mechanisms of NGF-induced hyperalgesia. Eur J Neurosci 1994;6:1903-12.

26. Andreev NYu, Dimitrieva N, Koltzenburg M, et al. Peripheral administration of nerve growth factor in the adult rat produces a thermal hyperalgesia that requires the presence of sympathetic post-ganglionic neurones. Pain 1995;63:109-15.

27. Dyck PJ, Peroutka S, Rask C, et al. Intradermal recombinant human nerve growth factor induces pressure allodynia and lowered heat-pain threshold in humans. Neurology 1997; 48:501-5

28. de Vries A, van Rijnsoever C, Engels F, et al. The role of sensory nerve endings in nerve growth factor-induced airway hyperresponsiveness to histamine in guinea-pigs. Br J Pharmacol 2001;134:771-6.

29. de Vries A, Engels F, Henricks PA, et al. Airway hyper-responsiveness in allergic asthma in guinea-pigs is mediated by nerve growth factor via the induction of substance P: a potential role for trkA. Clin Exp Allergy 2006;36:1192-200.

30. Bennedich Kahn L, Gustafsson LE, Olgart Höglund C. Nerve growth factor enhances neurokinin A-induced airway responses and exhaled nitric oxide via a histaminedependent mechanism. Pulm Pharmacol Ther 2008;21:522-32.
31. Nicol GD, Vasko MR. Unraveling the story of NGF-mediated sensitization of nociceptive sensory neurons: ON or OFF the Trks? Mol Interv 2007:7:26-41.

32. Nassenstein C, Schulte-Herbrüggen 0 , Renz $\mathrm{H}$, et al. Nerve growth factor: the central hub in the development of allergic asthma? Eur J Pharmacol 2006:533:195-206.

33. Guerios SD, Wang ZY, Boldon K, et al. Blockade of NGF and trk receptors inhibits increased peripheral mechanical sensitivity accompanying cystitis in rats. Am J Physiol Regul Integr Comp Physiol 2008:295:R111-22.

34. Ugolini G, Marinelli S, Covaceuszach $S$, et al. The function neutralizing anti-TrkA antibody MNAC 13 reduces inflammatory and neuropathic pain. Proc Natl Acad Sci USA 2007:104:2985-90.

35. Zhang YH, Nicol GD. NGF-mediated sensitization of the excitability of rat sensory neurons is prevented by a blocking antibody to the p75 neurotrophin receptor. Neurosci Lett 2004;366:187-92.

36. Davis JB, Gray J, Gunthorpe MJ, et al. Vanilloid receptor-1 is essential for inflammatory thermal hyperalgesia. Nature 2000:405:183-7.

37. Shu X, Mendell LM. Acute sensitization by NGF of the response of small-diameter sensory neurons to capsaicin. J Neurophysiol 2001;86:2931-8.

38. Stein AT, Ufret-Vincenty CA, Hua L, et al. Phosphoinositide 3-kinase binds to TRPV1 and mediates NGF-stimulated TRPV1 trafficking to the plasma membrane. J Gen Physiol 2006;128:509-22.

39. Zhu W, Oxford GS. Phosphoinositide-3-kinase and mitogen activated protein kinase signaling pathways mediate acute NGF sensitization of TRPV1. Mol Cell Neurosci 2007:34:689-700.

40. Chuang HH, Prescott ED, Kong H, et al. Bradykinin and nerve growth factor release the capsaicin receptor from Ptdlns(4,5) P2-mediated inhibition. Nature 2001:411:957-62.

41. Stempelj M, Ferjan I. Signaling pathway in nerve growth factor induced histamine release from rat mast cells. Inflamm Res 2005;54:344-9.

42. Hazari MS, Pan JH, Myers AC. Nerve growth factor acutely potentiates synaptic transmission in vitro and induces dendritic growth in vivo on adult neurons in airway parasympathetic ganglia. Am J Physiol Lung Cell Mol Physiol 2007;292:L992-1001.

43. Lambiase A, Bracci-Laudiero L, Bonini S, et al. Human CD4+ T cell clones produce and release nerve growth factor and express high-affinity nerve growth factor receptors. J Allergy Clin Immunol 1997;100:408-14.

44. Micera A, Vigneti E, Pickholtz D, et al. Nerve growth factor displays stimulatory effects on human skin and lung fibroblasts, demonstrating a direct role for this factor in tissue repair. Proc Natl Acad Sci USA 2001:98:6162-7.

45. Freund-Michel V, Bertrand C, Frossard N. TrkA signalling pathways in human airway smooth muscle cell proliferation. Cell Signal 2006;18:621-7.

46. Fang $\mathbf{X}$, Djouhri $\mathrm{L}$, McMullan $\mathrm{S}$, et al. trkA is expressed in nociceptive neurons and influences electrophysiological properties via Nav1.8 expression in rapidly conducting nociceptors. J Neurosci 2005:25:4868-78.

\section{The Dr H M (Bill) Foreman Memorial Fund}

The trustees of the above fund invite applications for grants relating to study in respiratory disease and allied fields (eg microbiology, histopathology, radiology, biochemistry and molecular biology). Limited funds are available for registered medical practitioners to assist in travelling to countries other than their own to study respiratory disease and also for support for clinical research abroad.

Intending applicants should write for further details to: Dr Brian H Davies, University Hospital Llandough, Penarth Vale of Glamorgan CF64 2XX, UK. 